\title{
Références bibliographiques du dossier : « le plaisir et l'ennui à l'école »
}

Bernadette Plumelle

\section{OpenEdition}

12 Journals

Édition électronique

URL : https://journals.openedition.org/ries/2113

DOI : 10.4000/ries.2113

ISSN : 2261-4265

Éditeur

France Education international

\section{Édition imprimée}

Date de publication : 1 septembre 2011

Pagination : 155-165

ISBN : 978-2-85420-592-3

ISSN : $1254-4590$

Référence électronique

Bernadette Plumelle, "Références bibliographiques du dossier : " le plaisir et l'ennui à l'école » », Revue internationale d'éducation de Sèvres [En ligne], 57 | septembre 2011, mis en ligne le 01 juillet 2012, consulté le 05 juillet 2021. URL : http://journals.openedition.org/ries/2113 ; DOI : https://doi.org/ $10.4000 /$ ries. 2113 


\title{
Références \\ bibliographiques
}

\section{Bernadette Plumelle}

\begin{abstract}
À l'heure où les systèmes éducatifs sont jugés sur leurs résultats, il existe un intérêt grandissant pour le rôle des émotions et de la motivation dans la réussite scolaire des élèves. L'affectivité à l'école, et plus spécifiquement l'ennui et son antidote, la motivation, ont été l'objet de nombreuses études ces dernières années.
\end{abstract}

Pour cette bibliographie non exhaustive, il a semblé pertinent de sélectionner des références récentes qui empruntent à différents champs d'étude : philosophie, sociologie, psychologie et psychanalyse, didactique et pédagogie. Les deux premières parties analysent, d'une part, le phénomène de la crise de la culture scolaire et les différentes philosophies de l'éducation sousjacentes; d'autre part, les travaux, d'ordre sociologique et psychanalytique, concernant le rapport au savoir.

Dans les parties centrales, sont rassemblées les références ayant pour sujet l'affectivité et l'école et plus précisément l'ennui et la motivation. Une dernière partie est axée sur des champs d'étude nouveaux pour l'apprentissage : les sciences cognitives et les pédagogies coopératives,

Les documents mentionnés sont pour la plupart consultables au Centre de ressources et d'ingénierie documentaires du CIEP ou accessibles sur Internet.

Bibliographie arrêtée le 4 juillet 2011.

\section{PHILOSOPHIE de L’Éducation ET CRISE DE LA CULTURE SCOLAIRE}

\author{
ARENDT Hannah, La crise de la culture : huit exercices de pensée politique, \\ Gallimard/Paris, 1989 [1972, première édition], 380 p.
}

L'ouvrage est un recueil d'articles extraits de diverses revues. Plusieurs textes ont pour objet la crise de l'éducation, et plus particulièrement de la crise qui traverse la structure de l'organisation de l'éducation et de ses principes dans la société américaine. Selon Hannah Arendt, l'école est ce qui permet la transition entre la famille et le monde, elle doit apprendre aux enfants ce qu'est le monde et non pas leur inculquer l'art d'y vivre. L'éducation sans enseignement n'est que rhétorique émotionnelle et morale.

\section{BLAIS Marie-Claude, GAUCHET Marcel, OTTAVI Dominique, Conditions de l'éducation, Stock/Paris, 2008, 265 p.}

Les auteurs proposent une réflexion sur ce qui pose problème aujourd'hui dans la transmission éducative. Toute une série de données qui servaient de socle à l'institution scolaire ont été ébranlées, voire sont en passe disparaître. Les auteurs mettent en lumière l'impact de ces transformations majeures et font ressortir le défi qu'elles représentent. L'analyse se développe sur quatre fronts : les relations de l'école avec la famille, le sens des savoirs dispensés, l'autorité dont l'école a besoin et sa place dans la société. L'ouvrage réserve une large place à la question du sens des savoirs scolaires. Dans le chapitre, "Des savoirs privés de sens? ", les auteurs considèrent que "la valeur intrinsèque de savoirs dispensés par l'école et l'évidence de les acquérir » n'est plus une valeur partagée, avec pour conséquence un sentiment d'ennui de la part des élèves, un rejet des savoirs et de l'institution scolaire. 


\section{BULLE Nathalie, L'école et son double : essai sur l'évolution pédagogique en France, Éditions Hermann/Paris, 2009, 324 p.}

Pourquoi le processus de démocratisation des systèmes éducatifs occidentaux a-t-il justifié un recours de plus en plus important à la pensée pédagogique dite moderne et un discrédit progressif de l'enseignement des disciplines, de leurs méthodes et de leurs contenus ? L'auteure montre que ces transformations ont suivi une voie définie par ceux qui entretiennent, par leur culture en sciences humaines, des représentations de l'homme et de son développement héritées du XIX ${ }^{e}$ siècle et fondées sur des bases fragiles ou fausses. Elle met au jour une série de croyances qui se sont constituées autour de l'école, de ses enseignements et ses méthodes, de ses succès comme de ses échecs.

\section{DEWEY John, Démocratie et éducation suivi de Expérience et éducation, Armand Colin/Paris, 2011, 520 p.}

Comment centrer l'éducation sur «les propres activités sociales de l'enfant » plutôt que sur les savoirs? Comment l'éducation peut-elle préparer l'enfant aux conditions sociales qu'il connaîtra hors de l'école ? John Dewey place l'expérience au cœur de l'apprentissage et recentre la pédagogie sur l'individu plutôt que sur les savoirs : l'école doit répondre à la curiosité naturelle de l'enfant et lui apprendre à désirer et entreprendre ; la motivation et l'effort individuel peuvent se substituer à la discipline et au régime de sanction. Ce volume propose la traduction des deux grands textes de John Dewey sur l'éducation ; son traité de pédagogie, Democracy and Education. An introduction to the Philosophy of Education (1916) et Experience and Education (1938) où l'auteur précise sa pensée et répond à ses détracteurs.

\section{JACQUET-FRANCILLON François, KAMBOUCHNER Denis (sous la direction de),} La crise de la culture scolaire : origines, interprétations, perspectives, PUF/ Paris, 2005, 505 p.

Issu d'un colloque organisé en 2003 à la Sorbonne, l'ouvrage réunit autour du thème de la crise de la culture scolaire un vaste ensemble de contributions de spécialistes dans les domaines tels que l'histoire, la sociologie, les sciences de l'éducation ou la philosophie. Les auteurs tentent, dans une approche diversifiée, de comprendre les causes de la crise scolaire qui affecte le système éducatif en France. Ils définissent ce concept en le reliant au phénomène beaucoup plus large des tensions qui secouent l'institution scolaire et aux bouleversements du système d'enseignement depuis quelques décennies. Enfin, ils élaborent des pistes de réflexion afin de construire une nouvelle philosophie appelant à une reconquête de la question culturelle à l'école.

\section{LOMBARD Jean, Hannah Arendt : Éducation et modernité, Éditions l'Harmattan/} Paris, 2003, $116 p$.

Dans les analyses qu'elle avait consacrées à la crise de l'école américaine dans les années soixante, Hannah Arendt avait diagnostiqué le caractère fatal de la subversion de l'éducation par la pédagogie et du transfert de l'autorité au monde enfant, qui ont gagné depuis tous les systèmes éducatifs occidentaux. Selon l'auteur, en définissant l'appareil conceptuel qui permet de penser l'école d'aujourd'hui, marquée par l'univers de la consommation, les phénomènes de masse, la perversion de la culture par le loisir, Hannah Arendt a édifié la philosophie de l'éducation de la modernité.

MEURET Denis, DESSBERG Laurent, "La discipline scolaire et le politique ", Penser l'éducation, juin 2006, $n^{\circ}$ 27, p. 21-38

Le thème de la discipline scolaire, développé par Durkheim dans L'Éducation morale, illustre la conception dominante au sein du modèle éducatif français. Cet article se propose de reprendre les dimensions psychologique et politiques de cette éducation, qui supposent aussi bien l'intériorisation de préceptes que la formation du citoyen, et de les confronter à la 
conception de la discipline défendue par Dewey, également soucieux de la croissance et de la formation du caractère des élèves. Si Durkheim conçoit la discipline comme une pratique collective censée rassembler les individus à l'image d'une société cohérente et ordonnée, Dewey promeut l'acquisition d'une aptitude à agir sans dissocier ses intérêts des buts poursuivis, afin d'enrichir, par voie de conséquence, les expériences sociales.

\section{MINOIS Georges, Les grands pédagogues : de Socrate aux cyberprofs, Éditions Audibert/Paris, 2006, 373 p.}

Les débats sur le rôle de l'école sont aussi vieux que l'école elle-même. Quelles valeurs doit-elle transmettre ? Et doit-elle privilégier l'enseignement culturel ou l'enseignement professionnel ? Depuis les premiers pédagogues de la Grèce ancienne, les éducateurs, précepteurs, instituteurs, professeurs, n'ont cessé de s'adapter tant bien que mal aux évolutions de la société. Cette synthèse replace dans leur contexte les idées de ceux qui ont fait progresser la réflexion pédagogique en Occident par leurs écrits ou par leurs pratiques : Socrate, Quintilien, Abélard, Érasme, Comenius, Locke, Rousseau, Pestalozzi, Durkheim, Montessori, Piaget, Freinet, et bien d'autres. Cette traversée historique de la question pédagogique de la Grèce ancienne à nos jours montre que la dynamique entre réforme et tradition est présente dès l'apparition de l'école.

OTTAVI Dominique, "Le défi d'enseigner aujourd'hui » in Crise du système scolaire ou crise de société ?, Revue de psychosociologie, janvier 2010, $n^{\circ} 9$, p. 13-27

Devant le sentiment d'une crise de l'éducation et la difficulté croissante d'enseigner, l'auteur tente de dépasser les constats pour analyser la crise des finalités de l'éducation dans notre culture. Les ravages des méthodes pédagogiques modernes sont souvent invoqués pour expliquer la crise et il faudrait revenir à des formes traditionnelles d'exercice de l'autorité et de transmission des savoirs. Selon l'auteur, c'est au niveau des principes de l'éducation que les difficultés doivent être analysées. Le système scolaire français accorde une place privilégiée à l'émancipation, sans en préciser le sens : s'agit-il de l'émancipation de l'individu par le savoir, de l'émancipation politique par la citoyenneté ou de l'émancipation de l'enfant?

\section{LE RAPPORT AU SAVOIR}

\section{ASTOLFI Jean-Pierre, La saveur des savoirs : disciplines et plaisir d'apprendre,} ESF/Paris, 2008, 252 p.

Cet ouvrage est consacré à l'examen, sous différentes facettes, des conditions qui rendent le savoir attrayant pour l'élève. L'auteur analyse ce qui peut pousser un élève à se mobiliser pour les savoirs et la satisfaction qu'il peut en tirer. Les quatre premiers chapitres problématisent la signification d'une école centrée sur la saveur des savoirs et réhabilitent l'idée des savoirs trop facilement déclarés académiques et inutiles. Les cinq autres chapitres, plus courts, reprennent cette problématique et l'appliquent à différents contextes.

\section{CHARLOT Bernard (sous la direction de), Les jeunes et le savoir : perspectives internationales, Anthropos/Paris, 2001, 168 p.}

Cette publication est le résultat d'un projet de recherche qui a rassemblé quatre pays : le Brésil, la France, la République tchèque et la Tunisie. Il s'agissait de confronter les sens que la notion de rapport au savoir reçoit et produit dans des aires culturelles diverses. Le livre est organisé en quatre temps. Tout d'abord, la notion de rapport au savoir est analysée avec ses points d'ancrage théoriques et ses fondements anthropologiques. Les deux chapitres suivants présentent un état des lieux de ce que les élèves brésiliens et tchèques disent avoir appris. L'analyse se poursuit, pour ces mêmes élèves, en situant leurs réponses en référence, pour les uns aux 
spécificités culturelles afro-brésiliennes, pour les autres à la philosophie grecque. Les derniers chapitres focalisent l'analyse sur des "objets didactiques", l'enseignement des sciences en Tunisie et l'enseignement de la philosophie et des sciences économiques en France.

COSNEFROY Laurent, "Rapport au savoir ", in Dictionnaire de l'éducation, VAN ZANTEN Agnès (sous la direction de), Presses universitaires de France/Paris, 2008, p. 597-599

Depuis la fin des années 1980, le rapport au savoir a été le fondement des travaux de deux équipes de recherche françaises, celle de Jacky Beillerot (CREF, Université de Paris X) et celle de Paris VIII avec Bernard Charlot (ESCOL). L'auteur propose une synthèse de leurs travaux, mettant en lumière les différences de conceptualisation. Pour les chercheurs du CREF, le rapport au savoir permet d'interroger les enjeux personnels, souvent inconscients, associés au savoir ou à un champ du savoir, avec la psychanalyse comme cadre théorique. Pour l'équipe de Bernard Charlot, le concept de rapport au savoir se définit comme rapport à des processus, à des produits et à des situations d'apprentissage.

HATCHUEL Françoise, Savoir, apprendre, transmettre : une approche psychanalytique du rapport au savoir, La Découverte/Paris, 2005, 158 p.

Que représente le savoir pour chacun et chacune d'entre nous? Que pensons-nous, ressentonsnous, vivons-nous lorsque nous savons, croyons savoir ou ne pas savoir, apprenons, essayons ou refusons d'apprendre, enseignons, produisons un savoir ? Depuis 1990, le collectif « Savoirs et rapport au savoir » de l'université Paris X Nanterre s'interroge sur les transformations de soi auxquelles doit faire face le sujet qui apprend.

MOSCONI Nicole, BEILLEROT Jacky, BLANCHARD-LAVILLE Claudine, et al. Formes et formations du rapport au savoir, Éditions l'Harmattan/Paris, 2000, $317 \mathrm{p}$.

Les auteurs de cet ouvrage appartiennent tous au CREF (Centre de Recherche Éducation et Formation) de Paris X Nanterre qui axe ses recherches sur la notion de savoir et de rapport au savoir. Les chercheurs ont organisé leur travail dans quatre directions : une direction théorique, où ils approfondissent les notions de savoir et de rapport au savoir, et trois directions de recherche empirique, où ils ont travaillé ces notions dans des champs spécifiques: les pratiques enseignantes, la formation des adultes, la biographie et l'autobiographie.

\title{
L'AFFECTIVITÉ À L'ÉCOLE : ENQUÊTES ET TÉMOIGNAGES DES ACTEURS
}

\author{
BLANCHARD-LAVILLE Claudine, Les enseignants entre plaisir et souffrance, \\ PUF/Paris, 2001, 281 p.
}

Plaisir et souffrance: c'est entre ces deux pôles qu'oscillent les enseignants dans l'exercice quotidien de leur pratique. Comment comprendre leurs difficultés ? L'auteure offre le fil rouge de son itinéraire personnel pour explorer l'univers enseignant au travers d'un récit vivant et sensible. Elle ouvre de nouvelles pistes pour accompagner les enseignants.

CAVET Agnès, Quelle vie scolaire pour les élèves?, Lyon: INRP/Cellule de veille scientifique et technologique, 2009, 20 p. téléchargées du site http://www.inrp.fr/

En France, depuis la loi d'orientation de 1989, l'élève est placé au centre du système éducatif. En vingt ans, sa place au sein de l'école a évolué : s'il est devenu un « usager » ayant des droits, l'école attend aussi de lui qu'il soit acteur de sa propre scolarité. Le système éducatif français 
va jusqu'à inclure la «vie scolaire » des élèves dans ses attributions. L'analyse porte sur la vie scolaire des élèves, leur cadre, la place offerte à leur expression et à leur participation, leur motivation et les valeurs qu'ils revendiquent dans l'espace scolaire.

CORNU Laurence, "La confiance ", Le Télémaque, février 2003, n²4, p. 21-30.

La confiance est un fait humain, et certaines de ses formes sont des relations et des actes où se joue une émancipation. Elle ne peut être l'objet ni d'un impératif moral, ni d'un impératif technique. Mais on en fait l'expérience et on peut chercher à penser son effectivité paradoxale, dans plusieurs approches (sociologique, éthico-politique, anthropologique, épistémologique) car elle est à l'œuvre, tout comme la méfiance, dans le lien social et transgénérationnel. Cette notion peut inspirer des réflexions décisives dans l'éducation et la formation des maîtres.

\section{DOUAT Etienne, L'école buissonnière: pour penser autrement l'absentéisme,} La Dispute/Paris, 2011, 208 p.

Qui sont ces élèves « absentéistes » que l'on rencontre pourtant si souvent dans leurs collèges? L'auteur rend compte d'une enquête qu'il a réalisée auprès de collégiens de deux collèges classés ZEP qui font l'école buissonnière. Ses investigations restituent et analysent l'oscillation de leurs pratiques et leur ambivalence face à l'institution scolaire, faites d'expériences socialisatrices contradictoires par rapport aux impératifs scolaires, aussi bien dans les familles ou les groupes de pairs, que dans les collèges eux-mêmes. Il s'attache à mettre en évidence le rapport des élèves et de leur famille à la « socialisation scolaire ».

ESPINOSA Gaëlle, L'affectivité à l'école, Presses universitaires de France/Paris, 2003, $233 p$.

Les élèves aiment-ils l'école? Aiment-ils apprendre? Aiment-ils leur professeur ? Qu'est ce qui fait l'essence même de la relation maître-élève? Cet ouvrage, se propose de mettre en valeur l'affectivité des élèves dans leur triple rapport à l'école, au savoir et au maitre, par le moyen d'entretiens auprès d'élèves en situation contrastée de réussite et d'échec. Le processus enseignement/apprentissage en situation scolaire a pour fin de permettre aux élèves de construire des savoirs, savoir-faire et savoir-être dont l'émergence ou non est appréciée en termes de réussite ou d'échec.

\section{GUMBEL Peter, On achève bien les écoliers, Paris/Grasset, 2010, 176 p.}

L'auteur, journaliste américain installé en France, apporte un regard critique sur la situation éducative en France. Dans cet essai, il donne un diagnostic sur les raisons des résultats peu satisfaisants de la France aux tests comparatifs internationaux et sur l'absence d'égalité des chances en fonction du milieu d'origine. Les enfants français sont dans l'ensemble plus anxieux et intimidés dans une salle de classe et davantage angoissés par la peur de l'échec; le système de notation comme les redoublements augmentent ces effets. Il examine certaines situations dans d'autres pays et, s'appuyant sur celles qui ont le plus de succès, conclut en proposant une série de pistes à suivre.

MARANDA Marie-France, VIVIERS Simon (sous la direction de), L'école en souffrance : psychodynamique du travail en milieu scolaire, Presses de l'université Laval/Québec, 2011, 192 p.

Cette enquête réalisée auprès du personnel scolaire d'une école secondaire révèle le désarroi et la détresse de celles et ceux qui, au quotidien, tentent d'accomplir la mission de l'école.

OCDE : organisation de coopération et de développement économiques, Résultats du PISA 2009 : apprendre à apprendre : les pratiques les stratégies et l'engagement des élèves - volume III, OCDE/Paris, 2011, 272 p.

Ce volume analyse la motivation des élèves de 15 ans, leur engagement envers la lecture et leur utilisation de stratégies d'apprentissage efficaces. Un chapitre analyse la relation positive entre 
l'engagement dans la lecture, l'approche à l'égard de l'apprentissage et la performance en compréhension de l'écrit. Il ressort des résultats que les élèves qui lisent par plaisir et qui font preuve de compétence lorsqu'ils ont à comprendre, mémoriser et synthétiser des informations complexes sont ceux qui obtiennent les meilleures performances. S'engager dans la lecture, adopter des stratégies efficaces d'apprentissage et devenir un lecteur compétent est un processus qui se nourrit de lui-même : les élèves qui lisent davantage deviennent de meilleurs lecteurs, et les bons lecteurs peuvent escompter de bons résultats en compréhension de l'écrit.

\section{PERRENOUD Philippe, Métier d'élève et sens du travail scolaire, ESF/Issy-les-} Moulineaux, 2000, 207 p.

Tout en proposant une analyse sociologique de l'éducation, ce livre est centré sur la vie quotidienne, les pratiques et les stratégies des élèves et des maîtres au sein de l'école. Il pose cette question récurrente : peut-on parler d'un métier d'élève ? L'auteur étudie ici ce "drôle de métier » et en explore les principales facettes : le rapport entre l'école et la vie, le curriculum formel face au curriculum réel (ensemble de l'expérience de l'élève), son rapport stratégique au travail scolaire et son comportement face à l'évaluation.

\section{POURTOIS Jean-Pierre, MOSCONI, Nicole (sous la direction de), Plaisir, souf- france, indifférence en éducation, PUF/Paris, 2002, 250 p.}

Les auteurs ont choisi une approche phénoménologique des notions de plaisir, de souffrance et d'indifférence. Des témoignages d'enfants et d'adultes ont été recueillis et leurs discours analysés par des experts d'horizons disciplinaires différents. À la fin de l'ouvrage, on trouve un article de Jean Houssaye "Vers des règles pédagogiques du plaisir et de la souffrance en éducation? ", un article de Pierre Mannoni «L'indifférence, un modèle de perversité éducative » et celui de Boris Cyrulnik «École. Une histoire naturelle de souffrance et de plaisir ».

SNYDERS Georges, J'ai voulu qu'apprendre soit une joie, Institut de recherches de la FSU/Les Lilas, Éditions Syllepse/Paris, 2008, 267 p.

Georges Snyders a été professeur de philosophie et de sciences de l'éducation. Son autobiographie permet de suivre le fil d'une vie et d'une pensée exigeante, à travers l'expérience d'Auschwitz, l'engagement politique et la réflexion pédagogique. Pour l'auteur, la question de la joie à l'école, le fait d'apprendre avec confiance et allégresse, est fondamentalement une question politique, puisque c'est celle de la possibilité même d'éduquer les citoyens.

\section{L'ennui à l'école et ses effets}

\section{BLAYA Catherine, Décrochages scolaires : l'école en difficulté, De Boeck Univer- sité/Bruxelles, 2010, 192 p. \\ L'ouvrage a pour objectif de présenter et d'analyser à partir de recherches en France et à l'étranger les conditions d'émergence du décrochage scolaire, tant au niveau individuel que familial, social et scolaire. L'approche du phénomène est multidisciplinaire et multifactorielle ; elle prend en compte les grandes « causalités » macro-sociales mais également l'effet établisse- ment, la relation à l'école et les capacités d'inclusion. Un chapitre est réservé aux facteurs scolaires et aux difficultés que l'école peut rencontrer dans la gestion du décrochage et de ses corollaires (absentéisme, échec scolaire, ennui). Les chapitres suivants analysent les politiques de lutte et de prévention contre le décrochage et les programmes d'intervention.}

CLERGET Joël, et al., Vivre l'ennui à l'école et ailleurs, Eres/Paris, 2006, 192 p.

L'ennui désigne à la fois une expérience individuelle et une figure du malaise social. Ordinaire ou pathologique, il apparaît comme une réalité incontournable et universelle qui interroge le rapport de l'homme à son désir et à son acte. Mais l'ennui est dévalorisé socialement et il n'est pas bien vu de s'ennuyer, surtout à l'école. L'ennui est pourtant tout autant le symptôme de l'institution que le signe du malaise de ceux qui la fréquentent, élèves et enseignants. Facteur 
de décrochage scolaire, il est cependant nécessaire à la transmission du savoir et fait partie intégrante du processus créatif. Issu d'une enquête auprès d'élèves du CM1 à la terminale dans les filières générales et professionnelles ainsi qu'auprès d'enseignants, ce livre à plusieurs voix envisage l'ennui dans tous ses états.

DURIF-VAREMBONT Jean-Pierre, CLERGET Joël et al. "L'ennui vu par les élèves : ses indicateurs et ses effets ", Connexions, 2005, $n^{\circ} 84$, p. 209-226

L'ennui se manifeste de plus en plus à l'école, mais l'école est l'occasion de cette manifestation, non la cause. Qu'en pensent les élèves eux-mêmes ? La parole a été donnée aux élèves et on a recueilli leur témoignage écrit sur leur vécu de l'ennui. L'analyse de contenu de 338 textes répartis selon le niveau scolaire et le sexe permet de distinguer les indicateurs et les effets (ou réactions) à l'ennui. Au-delà des variables, les élèves révèlent ainsi les processus et la structure de l'ennui.

\section{LELOUP Stéphanie, L'ennui des lycéens : du manque de motivation au décalage} des attentes, Thèse délivrée par l'université de Reims Champagne-Ardenne, 2003, mémoire de 618 p.

L'ennui est une notion extrêmement répandue dans le monde scolaire qui renvoie à des réalités très diverses comme l'ennui de l'enfant précoce ou celui qui ne comprend pas le cours. Pour toutes ces formes d'ennuis, on peut trouver une explication par le biais du " manque de motivation ». Cependant, la motivation est un processus psychologique, propre à un individu. Elle est difficilement mesurable et, les enseignants ont bien peu de prise sur ce phénomène. La thèse a pour objet la déconstruction du concept d'ennui en tant que "manque de motivation », et sa reconstruction en termes de "décalage d'attentes ». L'auteure a fondé son analyse à partir de trois types de données; un échantillon de questionnaires remplis par les lycées lors de la consultation nationale de 1998, des entretiens auprès des élèves et des enseignants.

OTTAVI Dominique, "Enfance et ennui contemporain ", La lettre de l'enfance et de l'adolescence, février 2005, $n^{\circ}$ 60, p. 49-54

L'ennui à l'école a toujours existé mais il est devenu récemment un sujet de préoccupation. Pour l'auteur « la chose n'est pas nouvelle mais il est nouveau que l'on en parle ». Il analyse le phénomène à partir d'un album de jeunesse, d'une gravure de Dürer et d'un roman. L'ennui a changé de nature à l'époque contemporaine; le temps scolaire et l'espace scolaire sont aujourd'hui générateurs d'un nouveau type d'ennui, d'une nouvelle difficulté à se projeter dans l'avenir.

VINCENT Jean-Didier, COMTE-SPONVILLE André, BERGOUNIOUX Pierre et al., L'ennui à l'école, Albin Michel - SCEREN/CNDP/Paris, 2003, 125 p.

Un colloque a été organisé à la Sorbonne par le Conseil national des programmes, "Culture scolaire et ennui ». Les différents contributeurs (neurobiologiste, philosophe, psychanalyste sociologue, enseignant, professeur en sciences de l'éducation...) apportent un regard croisé sur ce sentiment qu'on peut opposer au divertissement, intérêt, souffrance ou motivation. Après avoir défini la notion d'ennui, les auteurs se penchent sur les mythes et les réalités de l'ennui, et, enfin, sur les manières d'en faire bon usage.

\section{La motivation}

\section{DELANNOY Cécile, La motivation : désir de savoir décision d'apprendre,} Hachette Éducation - CNDP/Paris, 2005, 160 p.

L'auteure propose une lecture d'inspiration psychanalytique des racines du désir d'apprendre chez l'enfant et de la manière dont celui-ci opère au sein de la relation avec l'enseignant et le 
savoir dont il est porteur. Secondée par les réflexions du psychanalyste Jacques Lévine, elle met en rapport les structures du désir enfantin avec la question du sens des apprentissages, et montre comment le rapport au savoir s'établit en fonction d'une relation à soi et aux autres. Elle distingue trois types d'obstacles à ce désir d'apprendre déterminés par l'histoire du sujet. Et propose dans le dernier chapitre des pistes pratiques.

\section{GALAND Benoît, "Motivation ", in Dictionnaire de l'éducation, VAN ZANTEN} Agnès (sous la direction de), Presses universitaires de France/Paris, 2008, p. 485-489

La motivation est un ensemble de processus qui influencent l'engagement dans une activité : engagement affectif (intérêt, anxiété, satisfaction, ennui...), cognitif et comportemental. L'auteur offre un tour d'horizon des nombreuses recherches menées ces quinze dernières années sur les processus motivationnels. L'exposé s'organise autour de deux axes : les modèles théoriques qui s'attachent aux processus individuels, internes aux individus, et ceux qui s'attachent aux facteurs situationnels, internes aux contextes.

\section{GALAND Benoît, BOURGEOIS Etienne (sous la direction de), (Se) motiver à apprendre, PUF/Paris, 2006, 234 p.}

Ennui, absentéisme, décrochage scolaire, mais aussi épuisement professionnel et pénurie chez les enseignants... Les questions de motivation et d'engagement semblent être au cœur des défis qui se posent aujourd'hui aux acteurs de l'éducation. Comment lutter contre l'ennui en cours? Qu'est-ce que la motivation? Suffit-il de réussir pour être motivé ? Quelle est la place des relations sociales dans la motivation? Une vingtaine de chercheurs francophones font ici le point sur l'état des connaissances dans leur domaine et mettent en avant les implications pratiques de certains travaux novateurs. L'ouvrage, divisé en deux parties, présente de façon détaillée des travaux basés sur des théories mais qui visent à approfondir des questions plus précises en lien avec la pratique. La première partie est centrée sur la compréhension des processus motivationnels du point de vue de l'apprenant. La seconde partie est davantage axée sur l'analyse du rôle de l'environnement pédagogique, organisationnel et social dans la motivation.

\section{PINTRICH Paul, SCHUNK Dale, Motivation in education: theory, research, and application, Allyn \& Bacon/Boston, 2007 (troisième édition), 448 p.}

L'ouvrage a pour objectif principal de présenter les principales théories de la motivation ainsi que les principes et les résultats des recherches, en fournissant des exemples. Un chapitre introductif définit le concept de motivation ainsi que les principales théories de la motivation avec un éclairage historique. Les six chapitres suivants analysent les perspectives théoriques qui soulignent le rôle des cognitions personnelles, des croyances, des affects et des valeurs de la motivation. Les trois derniers chapitres portent sur divers facteurs contextuels favorables au renforcement de la motivation : les influences socioculturelles, les influences des enseignants, et les influences de la classe et de l'école.

RAICHE Gilles, NOEL-GAUDREAULT Monique (sous la direction de), "Motivation et réussite scolaire: dossier ", Revue des sciences de l'éducation, 2010, vol. XXXVI, $n^{\circ}$ 2, p. 321-468

Parmi une dizaine d'articles, deux travaux concernent spécifiquement la motivation. Le premier article retrace une recherche dont l'objectif était de vérifier si la motivation à apprendre et l'adaptation psychosociale se distinguent selon le sexe et la localisation socioéconomique de l'école. Elle a porté sur vingt-quatre écoles secondaires de divers milieux socioéconomiques. Les analyses montrent que l'école a moins d'attrait pour les élèves de milieu favorisé alors que les élèves de milieu défavorisé percevraient moins l'utilité de l'école. Le deuxième article est le résultat d'une recherche destinée à mesurer l'ampleur de l'effet de l'environnement scolaire sur la motivation et le rendement scolaire. 


\title{
VENTURINI Patrice, L'envie d'apprendre les sciences : motivation, attitudes rapport aux savoirs scientifiques, Fabert/Paris, 2007, $270 \mathrm{p}$.
}

La désaffection des études scientifiques est un phénomène préoccupant dans la mesure où sa persistance compromet à terme le développement économique des pays. Il pose question sur les pratiques scolaires existantes, sur les motivations à apprendre, sur le regard porté par les élèves sur les sciences et sur les processus qui facilitent ou engagent dans les filières scientifiques. L'auteur présente les travaux de recherche de psychologues, de scientifiques questionnant l'enseignement de leurs disciplines, de didacticiens ou de sociologues, menés depuis une vingtaine d'années dans un grand nombre de pays. Il décrit et analyse leurs fondements théoriques, les méthodologies utilisées ainsi que les résultats qu'ils ont permis.

\section{VIAU Rolland, La motivation en contexte scolaire, De Boeck Université/Bruxelles,} 1997, 220 p.

Après une définition de la motivation et de la place qu'elle occupe parmi les variables pédagogiques, l'auteur propose un modèle de motivation en contexte scolaire. Pour aider les enseignants à améliorer la motivation de leurs élèves, il propose une démarche en trois étapes : une réflexion sur les caractères des élèves, comment améliorer les activités d'enseignement et d'apprentissage pour susciter la motivation des élèves, enfin l'intervention directe sur les perceptions que l'élève a de lui-même et de ses processus d'apprentissage.

\section{NOUVELLES ORIENTATIONS, NOUVELLES PRATIQUES}

\author{
CARRE Philippe, L'apprenance: vers un nouveau rapport au savoir, Dunod/ \\ Paris, 2005, 224 p.
}

Économie du savoir, société cognitive, formation tout au long de la vie... L'acquisition de connaissances et de compétences nouvelles devient le levier majeur de l'adaptation et du développement des individus, des organisations et des nations du XXI ${ }^{\mathrm{e}}$ siècle. Le désir et la capacité d'apprendre sont en passe de devenir les compétences-clés. Pour l'auteur, la notion d'« apprenance ", " ensemble durable de dispositions favorables à l'acte d'apprendre dans toutes les situations ", illustre ce nouveau rapport au savoir : vouloir apprendre, savoir apprendre, pouvoir apprendre. L'ouvrage analyse ensuite trois conditions de son essor: comprendre les motivations à apprendre, développer les compétences d'autoformation, déployer une écologie de l'«apprenance».

\section{MORIN Edgar, La tête bien faite : repenser la réforme, réformer la pensée, Le Seuil/Paris, 1999, 160 p.}

L'inadéquation croissante entre les savoirs de plus en plus morcelés, l'hyperspécialisation, et la globalisation, l'interdépendance de problèmes culturels, sociologiques et politiques, impose une réforme de la pensée, donc de l'enseignement : "l'enseignement éducatif » transmettrait une culture qui permettrait de comprendre la condition humaine, favoriserait une pensée ouverte et libre, et non plus du pur savoir.

OCDE : organisation de coopération et de développement économiques, L'éducation un levier pour améliorer la santé et la cohésion sociale, OCDE/Paris, 2010, $245 p$.

À l'heure actuelle, le climat politique mondial souligne la nécessité de s'intéresser davantage aux composantes non-économiques du bien-être et du progrès social telles que la santé ou l'engagement social. L'éducation contribue largement à façonner ces indicateurs de progrès social. Ce rapport a pour objet d'apporter des éléments de réponse aux problèmes rencontrés 
dans l'évaluation des retombées sociales de l'éducation. À cet effet, il présente une synthèse des données probantes disponibles, passe en revue un certain nombre d'analyses de données antérieures et examine les débats politiques. La conclusion de ce rapport est que l'éducation peut contribuer à améliorer la santé et l'engagement civique et social et elle peut réduire les inégalités en développant les compétences cognitives, sociales et émotionnelles.

\section{Les sciences cognitives et l'apprentissage}

\section{DESSUS Philippe, GENTAZ Edouard, Apprentissages et enseignement : sciences cognitives et éducation, Dunod/Paris, 2006, 247 p.}

Quels sont les processus cognitifs et sensori-moteurs sous-jacents à l'apprentissage de l'écriture ? Quelles sont les méthodes pédagogiques favorisant la compréhension en lecture ? Quels sont les différents types de motivation scolaire, leurs origines et leurs conséquences sur l'apprentissage? L'ouvrage a pour objet d'analyser les processus mis en œuvre par les élèves lorsqu'ils apprennent et les enseignants lorsqu'ils enseignent. Il comprend treize chapitres, coécrits par une trentaine de chercheurs et enseignants-chercheurs en psychologie, sciences de l'éducation, technologie de l'information et de la communication et sciences et techniques des activités physiques et sportives.

DONOVAN Suzanne, BRANSFORD John D. (ed.), How students learn: history, mathematics, and science in the classroom, National academies Press/Washington, 2005, 615 p.

Après avoir publié un ouvrage, d'ordre théorique, intitulé How people research? en 1996, le Comité sur l'apprentissage du National Research Council (NRC) a pour objet, avec ce volume, de mettre en pratique les résultats de recherches sur la cognition, l'enseignement et l'apprentissage. Le livre explore comment de nouvelles stratégies d'apprentissage peuvent être appliqués dans la classe. Elles portent sur trois disciplines; l'histoire, les sciences et les mathématiques et à des niveaux différents.

\section{DUMONT Hanna ed., ISTANCE David ed., BENAVIDES Francisco ed., Comment apprend-on? La recherche au service de la pratique, OCDE/Paris, 2010, 356 p.}

Que savons-nous de la façon dont nous apprenons? Quelle influence les motivations et les émotions des jeunes ont-elles sur leurs apprentissages? Dans quelles circonstances le travail en groupe, l'évaluation formative ou les pédagogies par projet donnent-ils les meilleurs résultats? Qu'en est-il de l'apprentissage à l'aide des technologies ou au sein de la famille? Les auteurs ont pour objectif d'établir un pont entre la recherche sur l'apprentissage des élèves et le monde des politiques publiques et des pratiques d'autre part. Les contributions de chercheurs d'Amérique du Nord et d'Europe apportent des éclairages sur la nature de l'apprentissage et analysent quelles peuvent être les implications pour la conception de l'enseignement. Un chapitre, intitulé "Motivation et émotion : deux piliers de l'apprentissage en classe » fait la synthèse des connaissances sur l'importance du rôle des émotions et de la motivation et en dégage un ensemble de principes clés.

\section{LENA Pierre, AJCHENBAUM-BOFFETY Béatrice (coord.), Éducation sciences cognitives et neurosciences : quelques réflexions sur l'acte d'apprendre, Presses Universitaires de France/Paris, 2008, 226 p. \\ Qui ne s'interroge aujourd'hui sur les meilleures manières d'enseigner? Depuis la décennie 1990, les neurosciences cognitives et la compréhension du cerveau connaissent une expansion consi- dérable et éclairent les processus d'apprentissage. L'Académie des sciences, en collaboration avec}


le Centre pour la recherche et l'innovation dans l'enseignement (CERI) de l'OCDE, a organisé, en 2005, un séminaire sur le thème "Éducation, cognition et cerveau ", associant des spécialistes de neurosciences. Que nous apprend aujourd'hui l'investigation directe du cerveau ? Quels éclairages apporte-t-elle sur l'acte d'apprendre, qu'il s'agisse de la lecture, des langues, de la mémoire ou du calcul ? Voici quelques-unes des questions abordées au cours de cette rencontre.

\title{
Approches pédagogiques
}

\begin{abstract}
ALTET Marguerite, Les pédagogies de l'apprentissage, PUF/Paris, 2006 [deuxième édition], $128 \mathrm{p}$.

Une analyse des classifications récentes des différentes pédagogies permet de dégager un courant contemporain, basé sur l'apprentissage, où l'on retrouve des pédagogies aux caractéristiques communes : conception constructiviste de l'apprentissage, centrage sur l'activité de l'apprenant dans sa construction du savoir, instrumentation des moyens d'apprendre, développement des stratégies métacognitives. Ces pédagogies sont décrites, avec l'appui de textes de précurseurs et de représentants contemporains.
\end{abstract}

\section{CONNAC Sylvain, Apprendre avec les pédagogies coopératives : démarches et outils pour l'école, ESF/Paris, 2009, 334 p.}

L'ouvrage se présente comme un manuel de pédagogie pour l'école primaire avec un ensemble complet d'outils couvrant aussi bien les objectifs d'apprentissage que l'organisation de la classe, la construction de l'autonomie ou l'accès à la pensée réflexive. Les apports théoriques et les exemples concrets s'y nourrissent réciproquement, Des références historiques, des expériences de classes et d'école, des tableaux synthétiques, un lexique complet des principales notions complètent ce livre.

MUSSET Marie, THIBERT Rémi, Quelles relations entre jeu et apprentissages à l'école? Une question renouvelée, INRP/Cellule de veille scientifique et technologique/Lyon, 2009, 15 p. téléchargées du site http://www.inrp.fr/

De nombreux travaux de recherche éclairent la place et le rôle du jeu dans le processus d'apprentissage : impact positif sur la motivation et les apprentissages des élèves, l'acquisition de connaissances et le développement de compétences. Des initiatives, individuelles ou institutionnelles, ont été prises dans différents systèmes éducatifs. Le dossier propose d'explorer les relations entre le jeu et l'école à travers une revue de la littérature scientifique récente, en France et à l'étranger.

ROUILLER Yviane, LEHRAUS Katia (ed.), Vers des apprentissages en coopération : rencontres et perspectives, Peter Lang/Berne, 2008, 237 p.

L'ouvrage regroupe des contributions offrant une pluralité d'éléments pour prendre conscience des enjeux de la coopération, de ses caractéristiques, de ses exigences, de ses variations et de ses influences. Il permet de mieux comprendre les similitudes et les différences entre des approches venant de l'Europe francophone et celles issues d'Amérique du nord. Après une introduction qui rappelle les deux ancrages théoriques fondateurs des approches coopératives : les travaux de Piaget et ceux de Vygotski, deux grandes parties offrent un panorama de recherches et de réflexions. L'ouvrage se termine par un essai de "modélisation des conditions favorables aux apprentissages coopératifs » insistant sur la nécessaire alliance entre le social, le cognitif et l'affectif dans la coopération. 